\title{
BIBLIOGRAPHIE
}

[1] P. Macherel. Le Lait, 1948, 28, 256.

[2] J. PIEN. Le Eait, 1948, 28, 225.

[3] D. Florentin. Le Lait, 1939, 19, 10.

[4] M. BenNaerts. Nederl. Melk-Zuiveltijdschrift, 1948, 2, 99.

[5] L. C. JANSE. Methods of fat determination in dairy products. Chem. Weekblad, 1946, 42.

\section{INFLUENCE DES ALBUMINES SUR LA STABILITÉ DU BEURRE (1)}

\author{
par

\section{P. DIATCHENKO}

(Institut des recherches scientifiques de l'Industrie Laitière de l'U.R.S.S.)

Dans un article antérieur (Industrie Laitière, avril 1949) sur la teneur en albumines et la composition du plasma du beurre, nous avons montré que le goût et l'odeur du beurre dépendent dans une certaine mesure des propriétés du plasma.

Les albumines du plasma pendant leur conservation peuvent subir sous l'influence des microorganismes, une certaine dégradation. Celle-ci peut être la cause des défauts du gout du beurre. Le but de nos recherches est d'élucider cette question.

Dans les essais de laboratoire le plasma a été extrait du beurre de vache frais. Le liquide a été ensemencé ensuite par de différentes bactéries. Après un séjour de cinq jours dans un thermostat, on examinait le goût du plasma et déterminait le taux de dégradation des albumines en dosant l'azote aminé. Le tableau I montre que la décomposition des albumines du plasma détermine l'apparition des défauts suivants : goût amer, putrescent, etc.

Il est à signaler notamment que le goût dit " de poisson " apparaît dans le plasma lors d'une putréfaction profonde. Ceci est en contradiction avee l'explication admise généralement, selon laquelle l'origine de ce défaut serait le processus catalytique de décomposition de la lécithine sous l'action du sel et de l'acide lactique avec formation de la triméthýlamine: Nos essais ont montré que ce défaut peut apparaître lors de la dégradation microbiologique du complexe albumino-lécithinique des enveloppes des particules sphériques de la graisse.

(1) Industrie Laitière (de 1'U.R.S.S.), juillet 1949, p. 17-20. (Tracluction C. Barkovsky.) 
TABLEAU I

\begin{tabular}{|c|c|c|}
\hline Nature des bactéries & $\begin{array}{l}\text { Accroissement } \\
\text { de l'azote aminé }\end{array}$ & Défaut de goût observé \\
\hline Première série & & \\
\hline Mammocoques .............. & 16 & Amer \\
\hline Putréfactives .............. & 94 & De poisson (hareng) \\
\hline Spores (bâtonnet de) ............ & 7 & Très amer \\
\hline Streptocoques.............. & 4 & D'acide lactique \\
\hline Bâtonnet mobile.............. & 36 & Putrescent \\
\hline Bâtonnet d'acide lactique ...... & 6 & D'aeide lactique \\
\hline Deuxième série & & \\
\hline Mammoeoque $\ldots \ldots \ldots \ldots \ldots \ldots$ & 15 & Amer, putrescent \\
\hline Putréfactives . . . . . . . . . . . & 11 & De poisson (hareng) \\
\hline Mycoderma...$\ldots \ldots \ldots \ldots \ldots$ & 8 & Putrescent \\
\hline Culture lactique $\ldots \ldots \ldots \ldots \ldots \ldots$ & 7 & D'acide lactique \\
\hline Troisième série & & \\
\hline Streptocoques............. & 2 & D'acide lactique \\
\hline Levure de lait $\ldots \ldots \ldots \ldots \ldots \ldots$ & 1 & Alcoolique \\
\hline Spores (bâtonnet de $\rightarrow$ ) $\ldots \ldots \ldots$ & 6 & Très amer \\
\hline
\end{tabular}

Dans une autre série d'essais nous avons étudié la dégradation des albumines dans le beurre lui-même. Les échantillons de beurre étaient préparés à partir de la crème additionnée de différentes bactéries putréfactives. L'échantillon de référence était préparé avee de la crème ensemencée avec des bactéries lactiques.

Le tableau II nous montre que la dégradation des albumines sous l'influence des bactéries putréfactives se manifeste également dans le beurre de vache (après un séjour de 45 jours à la température de $\left.+7^{\circ}\right)$. L'échantillon de référence n'a accusé qu'un taux insignifiant de dégradation des albumines sans apparition de défauts de goût.

Ces essais de laboratoire montrent que le processus de dégradation des albumines dans le beurre lui-même progresse beaucoup moins rapidement que dans le plasma isolé. Ceci s'explique par la structure même du beurre et notamment par la distribution du plasma dans le beurre et par la grosseur de ses gouttelettes. D'après les travaux de Titoff (voir Travaux de l'Inst. Sci. de l'Industrie Laitière, 1947) - le degré élevé de dispersion du plasma dans le beurre détermine la grande stabilité de celui-ci. Titoff a trouvé que le beurre le plus stable à la conservation est celui dans lequel 
le plasma se trouve sous forme de gouttelettes de dimensions inférieures à celles des bactéries.

\section{TABLEAU II}

\begin{tabular}{|c|c|c|}
\hline Nature des bactéries & $\begin{array}{l}\text { Accroissement } \\
\text { de l'azote aminé }\end{array}$ & Défaut du goût observé \\
\hline Première série & & 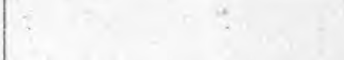 \\
\hline Echantillón de référence $\ldots . \ldots \ldots$ & 0 & Désagréable : vieux \\
\hline $\begin{array}{c}\text { Streptocoques (acide lactique) .... } \\
\text { Deuxième série }\end{array}$ & 1 & Fade \\
\hline $\begin{array}{l}\text { Echantillon de référence ......... } \\
\text { Inoculation lactique du beurre } \ldots\end{array}$ & $\begin{array}{l}1 \\
2\end{array}$ & $\begin{array}{l}\text { Légèrement amer } \\
\text { Aigre, "graisseux " }\end{array}$ \\
\hline Troisiême série & & \\
\hline Echantillon de référence . . . . . . . & 1 & Légèrement désagréable \\
\hline Putréfactives .............. & 4 & Vieux, de fromage \\
\hline Putréfactives . ............. & -21 & Amer, de fromage \\
\hline Putréfactives .............. & 9 & Rance \\
\hline Putréfactives ............... & 6 & Vieux \\
\hline Quatrième série & & \\
\hline Echantillon de référence $\ldots \ldots \ldots$ & 2 & Impur \\
\hline Streptocoque (acide lactique) .... & 3 & Aigre \\
\hline Spores (bâtonnet de $\rightarrow$ ) ....... & 9 & Rance \\
\hline Mammocoques $\ldots \ldots \ldots \ldots \ldots \ldots$ & 11 & Amer \\
\hline Micoderma $\ldots \ldots \ldots \ldots \ldots \ldots$ & 16 & Fermenté \\
\hline
\end{tabular}

Notons que dans les échantillons courants de beurre le plasma est dispersé sous forme de gouttelettes de grosseurs variées.

En vue d'étudier la dégradation des albumines du plasma du beurre laitier ordinaire nous nous sommes adressé à la "base laitière " de Tchérepovets (Dép-t de Vologda) qui nous a fait parvenir des échantillons de ses différents beurres (beurre de Vologda, beurre doux, beurre " acide» salé et non salé). Les essais de conservation (5 mois) s'effectuaient à la température de $0^{\circ}$ à $+7^{\circ}$. Au cours de cette conservation on procédait périodiquement à l'expertise du goût et à l'analyse quantitative de l'azote aminé. Le tableau III résume les résultats de ces essais.

En se basant sur les taux d'accroissement de l'azote aminé et sur la diminution des coefficients du goût et de l'odeur du beurre les différents échantillons peuvent être classés en trois séries (groupes) : 
TABLEAU III

\begin{tabular}{|c|c|c|c|c|c|c|c|c|c|}
\hline \multirow[b]{2}{*}{$\begin{array}{l}\text { No de } \\
\text { l'usine }\end{array}$} & \multirow[b]{2}{*}{ Nature du beurre } & \multirow[b]{2}{*}{ 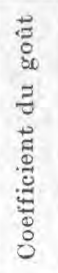 } & \multirow[b]{2}{*}{$\begin{array}{c}\% \\
\text { d'eau }\end{array}$} & \multirow[b]{2}{*}{$\begin{array}{r}\% \\
\text { du } \\
\text { sel }\end{array}$} & \multicolumn{3}{|c|}{ Dans le plasma. } & \multicolumn{2}{|c|}{ Après 150 jours } \\
\hline & & & & & $\begin{array}{c}\% \\
\text { d'albu- } \\
\text { mine }\end{array}$ & $\begin{array}{c}\% \\
\text { du } \\
\text { sucre } \\
\text { de lait }\end{array}$ & $\begin{array}{c}\text { Beurre } \\
\text { Coeffi- } \\
\text { cient } \\
\text { du } \\
\text { goût }\end{array}$ & $\begin{array}{c}\text { Plasma } \\
\text { Accroisse- } \\
\text { ment d'N } \\
\text { aminé } \\
\text { en degrés }\end{array}$ & $\begin{array}{c}\text { Coeffì } \\
\text { eient } \\
\text { du goût }\end{array}$ \\
\hline & $\begin{array}{c}\text { Première série } \\
(+)\end{array}$ & & & & & & & & \\
\hline 361 & Beurre " acide " non salé .. & 41 & 16,0 & - & 2,34 & 2,29 & 39 & 0 & 41 \\
\hline 321 & Beurre " acide $"$ salé. . . . . . . & 42 & 15,5 & 1,0 & 2,53 & 2,44 & 40 & 0 & 43 \\
\hline 204 & Beurre « acide salé $\ldots . . \ldots$ & 43 & 15,0 & 1,1 & 1,85 & 2,17 & 37 & 0 & 41 \\
\hline 17 & Beurre doux non salé...... & 42 & 16,0 & - & 2,30 & 2,21 & 37 & $+0,5$ & - \\
\hline 204 & Beurre " acide " salé. . . . . . & 41 & 15,7 & 0,9 & 1,79 & 3,01 & 38 & - & - \\
\hline 323 & Beurre "s acide "salé....... & 37 & 15,8 & 1,3 & 2,32 & 3,32 & 36 & 0 & 38 \\
\hline & Deuxième série & & & & & & & & \\
\hline 394 & Beurre doux salé ........ & 41 & 15,2 & 1,3 & 1,93 & 3,14 & 40 & $+1,5$ & 44 \\
\hline 20 & Beurre de Vologda $(+) \ldots$ & 45 & 15,0 & - & 4,86 & 4,72 & 39 & $+8,5$ & 38 \\
\hline 20 & Beurre de Vologda....... & 44 & 15,2 & - & 4,97 & 4,45 & 39 & +9 & 37 \\
\hline 22 & Beurre " acide " sale. . . . . . . & 42 & 15,5 & 1,0 & 1,66 & 2,75 & 39 & +2 & 38 \\
\hline 321 & Beurre " acide "salé . . . . . . & 41 & 15,6 & 1,0 & 2,53 & 2,29 & 39 & $+2,5$ & 42 \\
\hline 323 & Beurre " acide " salé. . . . . . . & 44 & 14,9 & 1,3 & 2,22 & 2,48 & 37 & $+4,2$ & 39 \\
\hline 7 & Beurre doux salé ......... & 42 & 15,5 & 1,0 & 2,24 & 2,80 & 37 & $+3,7$ & 36 \\
\hline 40 & Beurre de Vologda........ & 45 & 15,6 & - & 4,06 & 4,52 & 39 & $+3,7$ & 37 \\
\hline 204 & Beurre "acide» salé. . . . . . & 43 & 15,7 & 1,0 & 1,48 & 2,77 & 37 & +2 & 36 \\
\hline & Troisième série & & & & & & & & \\
\hline 1 & Beurre de Vologda........ & 43 & 15,0 & - & 4,01 & 3,44 & 42 & $+0,7$ & 44 \\
\hline, 1 & Beurre de Vologda........ & 43 & 15,6 & - & 4,37 & 4,41 & 41 & $+0,5$ & 44 \\
\hline 20 & Beurre doux non salé ..... & 42 & 15,2 & - & 2,83 & 3,88 & 41 & $+0,5$ & 44 \\
\hline 17 & Beurre doux non salé .... & 42 & 14,6 & - & 2,28 & 3,14 & 41 & 0 & 44 \\
\hline 17 & Beurre doux non salé ..... & 40 & 15,6 & - & 2,0 & 2,57 & 41 & 0 & 43 \\
\hline 17 & Beurre doux non salé ..... & 42 & 15,2 & - & 2,85 & 2,32 & 41 & $+0,7$ & 38 \\
\hline 7 & Beurre doux sàlé ......... & 43 & 15,2 & 1,0 & 3,25 & 3,16 & 42 & 0 & 44 \\
\hline 7 & Beurre doux salé ........ & 42 & 15,4 & 1,0 & 2,52 & 2,34 & 42 & - & 44 \\
\hline 301 & Beurre doux salé ......... & 43 & 15,4 & 1,1 & 2,39 & 2,78 & 42 & $+0,5$ & 44 \\
\hline 321 & Beurre " acide» salé....... & 40 & 15,5 & 1,0 & 2,44 & 3,15 & 41 & +05 & 44 \\
\hline
\end{tabular}

(t) Pour la définition de ces différentes qualités du beurre, voir les ouvrages de Sirik ou de Davidoff.

Première série : beurre instable, dont le coefficient du goût et de l'odeur est inférieur à 41. Pas de phénomène de dégradation d'albumines.

Deuxième série : beurre instable, dont le coefficient du goût et 
de l'odeur est inférieur à 41. La dégradation des albumines y est prononcée.

Troisième série : beurre stàble, dont le coefficient du goût et de l'odeur est supérieur à 41 , donc le coefficient d'un beurre de qualité supérieure. Pas de phénomène de dégradation d'albumine et de graisse.

Notons les constatations suivantes : les albumines n'ont joué aucun rôle dans la mauvaise stabilité des échantillons de la première série. Les échantillons de la deuxième série accusèrent un accroissement de taux d'azote aminé de plus de $2^{\circ}$. La dégradation profonde des albumines sous l'influence des bactéries putréfactives a déterminé des défauts considérables du goût du beurre. Cependant la diminution accentuée des coefficients du goût du plasma et même des matières grasses du lait montre que la dégradation des albumines du plasma est liée à d'autres phénomènes déterminant les défauts du beurre par décomposition du sucre de lait et des matières grasses. Ainsi done la stabilité du beurre à la conservation dépend en grande partie des phénomènes de putréfaction qui y prennent naissance.

L'analyse bactériologique du beurre, en vue de la détermination des bactéries putréfactives, est assez complexe et ne peut, par conséquent, être effectuée dans toutes les laiteries. Nous avons élaboré une méthode simple et pratique permettant de conduire cette analyse dans chaque laiterie.

La méthode de laboratoire de détermination du degré de putréfaction du babeurre est basée sur un double titrage au formol. On prélève de la baratte deux prises d'essais d'au moins $125 \mathrm{~cm}^{3}$ chacune. L'une est introduite dans un petit flacon ou un tube à essais propre et rincé au préalable à l'eau bouillante. On ferme au moyen d'un tampon de coton sec et abandonne dans un endroit frais pendant au moins sept jours. Dans les deux échantillons (babeurre frais et babeurre ayant séjourné au frais) on détermine l'acidité par la méthode usuelle et l'azote aminé par titrage au formol. Pour ce dernier titrage on ajoute à la prise d'essai, après titrage de l'acidité, $1 \mathrm{~cm}^{3}$ de formol neutralisé à la phtaléine du phénol jusqu'à la coloration rose pâle. Après addition du formol la solution incolore est additionnée de soude jusqu'à l'apparition de la coloration rose pâle. La quantité de soude décinormale consommée pour le titrage de $10 \mathrm{~cm}^{3}$ de babeurre en présence de formol est multipliée par 10, on obtient ainsi la quantité, en degrés, d'azote aminé. La différence entre le degré d'azote aminé trouvé dans les deux échantillons représente l'accroissement du taux de l'azote aminé dans l'échantillon de babeurre ayant séjourné au frais pendant unẹ 
semaine et indique par conséquent la dégradation des albumines produite par la putréfaction.

Il serait souhaitable de contrôler ce procédé dans de plus larges limites de durée de conservation (5 à 10 jours) et de températures y compris celles des chambres frigorifiques des laiteries.

Dès que les données expérimentales seront en nombre suffisant, on pourra établir une échelle d'appréciation de l'état sanitairobactériologique de la production dans chaque laiterie donnée, ceci d'après le taux de la contamination du babeurre par des microorganismes putréfactifs. La présence de ces microorganismes dans le babeurre peut servir d'indication indirecte sur la présence des bactéries putréfactives dans le beurre.

Cette méthode peut servir également pour déceler dans l'eau la présence des bactéries putréfactives. Dans ce cas on analysera le babeurre dilué de deux fois son volume d'eau à analyser.

Le groupe des beurres stables à la conservation (troisième série du tableau III) est d'un intérêt tout particulier pour notre étude. On remarque notamment que toutes les variétés du beurre (beurre de Vologda, beurre doux, beurre "acide ») y figurent. Elles contiennent des quantités différentes d'albumines dans le plasma, mais sont exemptes de bactéries putréfactives.

Les résultats de nos essais permettent donc de considérer comme établi le fait que la stabilité du beurre à la conservation ne dépend pas de la quantité d'albumines qu'il contient. Les conditions fondamentales de sa stabilité sont les suivantes : utilisation du lait frais et de la crème fraîche et l'observation stricte du régime sanitaire-hygiénique. Le beurre de Vologda est le plus caractéristique à ce point de vue, il contient en effet plus d'albumines que les autres et ceci pour tous les échantillons reçus de différentes laiteries. Ainsi l'échantillon de l'usine $n^{0} 1$ possède une grande stabilité, tandis que les échantillons des usines no 20 et no 40 (deuxième série) sont instables pour cause de la dégradation des albumines sous l'action des bactéries putréfactives.

Le beurre le plus remarquable par sa stabilité (même aux températures supérieures à $\left.0^{\circ}\right)$ est celui de la laiterie d'Eliakhine ( $\left.n^{\circ} 7\right)$. Les échantillons de ce beurre en plus de leur grande stabilité à la conservation possédèrent une propriété spéciale : stabilité à la moisissure. Ajoutons que les échantillons des laiteries de Partenoff (n० 394) et de V..-Selsky ( ${ }^{\circ} 321$ ) se sont montrés également résistants à la moisissure. 